\title{
Measurement of the Position-Dependent Electrophoretic Force on DNA in a Glass Nanocapillary
}

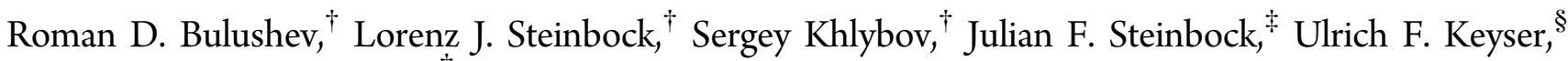 \\ and Aleksandra Radenovic ${ }^{* \dagger}$ \\ ${ }^{\dagger}$ Laboratory of Nanoscale Biology, Institute of Bioengineering, School of Engineering, EPFL, 1015 Lausanne, Switzerland \\ ${ }^{\ddagger}$ Faculty of Physics, Technical University of Kaiserslautern, 67663 Kaiserslautern, Germany \\ ${ }^{\S}$ Cavendish Laboratory, University of Cambridge, Cambridge CB3 0HE, United Kingdom
}

\section{Supporting Information}

\begin{abstract}
The electrophoretic force on a single DNA molecule inside a glass nanocapillary depends on the opening size and varies with the distance along the symmetrical axis of the nanocapillary. Using optical tweezers and DNA-coated beads, we measured the stalling forces and mapped the position-dependent force profiles acting on DNA inside nanocapillaries of different sizes. We showed that the stalling force is higher in nanocapillaries of smaller diameters. The position-dependent force profiles strongly depend on the size
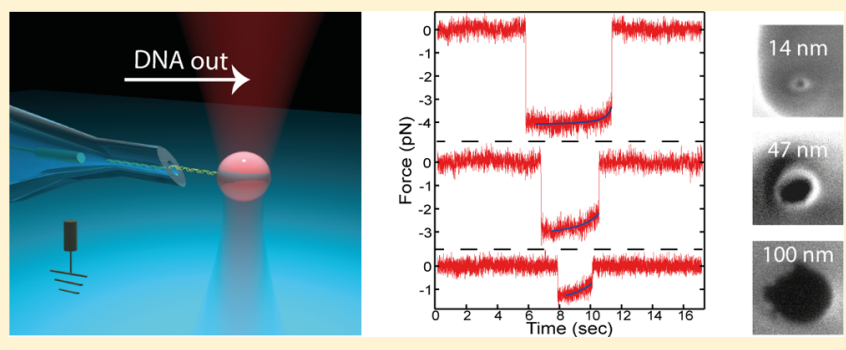
of the nanocapillary opening, and for openings smaller than 20 $\mathrm{nm}$, the profiles resemble the behavior observed in solid-state nanopores. To characterize the position-dependent force profiles in nanocapillaries of different sizes, we used a model that combines information from both analytical approximations and numerical calculations.
\end{abstract}

KEYWORDS: Single molecule measurements, nanocapillary, solid-state nanopore, DNA translocation, optical tweezers, force measurements

S olid-state nanopores are label-free sensing platforms for the detailed characterization of single molecules. They have found applications as detectors of DNA, ${ }^{1}$ RNA, ${ }^{2}$ proteins, ${ }^{3}$ and DNA-protein complexes ${ }^{4}$ and are currently promising candidates for next generation DNA sequencing. ${ }^{5,6}$ Furthermore, when combined with optical tweezers, solid-state nanopores can answer fundamental questions by revealing single molecule mechanisms. This technique was first applied to measure the charge of DNA in solution. ${ }^{7}$ Later, Van Dorp et al. used optical tweezers in combination with nanopores to estimate the impact of electroosmotic flow during translocation of DNA molecules through nanopores of different sizes. ${ }^{8}$ Biological applications of this method include calculation of the charge of single RNA molecules ${ }^{9}$ and DNA-protein complexes ${ }^{10}$ and the detection of single proteins bound to DNA. ${ }^{11,12}$

To produce a classical solid-state nanopore, first a $\mathrm{Si}_{3} \mathrm{~N}_{4}$ or $\mathrm{SiO}_{2}$ membrane is fabricated using lithography techniques. Next, a pore of desired diameter is drilled by transmission electron microscopy (TEM) ${ }^{13}$ or focused-ion beam (FIB). ${ }^{14} \mathrm{~A}$ cheaper and faster alternative to nanopore fabrication is laser pulling of glass capillaries, a technique that does not require cleanroom facilities. This process results in nanocapillaries with openings as small as $50 \mathrm{~nm} .{ }^{15}$ Similar to solid-state nanopores, glass nanocapillaries can detect single molecules of $\mathrm{DNA}^{15}$ and proteins. ${ }^{16}$ They can also be combined with optical tweezers for ultrasensitive force measurement experiments. ${ }^{17,18}$ In this combination, nanocapillaries have advantages over nanopores due to a simpler design of the microfluidic cell, more sensitive lateral displacement of the optically trapped bead during DNA capturing, and easier determination of the proximal location of the pore. Keyser et al. used this setup in a variety of applications including studying the mechanism of DNA relaxation, ${ }^{19}$ investigating the behavior of charged polymers in crowded environments, ${ }^{20}$ and measuring electroosmotic nanojets. ${ }^{21}$

The development of a fast and simple method to control the geometry of the nanocapillary opening under SEM irradiation represents a new step in nanocapillary-based sensing. ${ }^{22}$ Melting the glass by exposing it to an electron beam leads to shrinking of the opening and changing of the shape of the tip. Most importantly, it results in a geometry that resembles membraneembedded nanopores. Shrunken nanocapillaries demonstrate higher drops in conductance during DNA translocation when compared to nonshrunken ones. ${ }^{23}$ The high signal-to-noise ratio of glass nanopores allows for the detection of short DNA molecules, proteins as small as $12 \mathrm{kDa}$, and DNA-protein complexes. $^{24}$ Thus, glass nanocapillaries have been established

Received: August 26, 2014

Revised: October 21, 2014

Published: October 24, 2014 

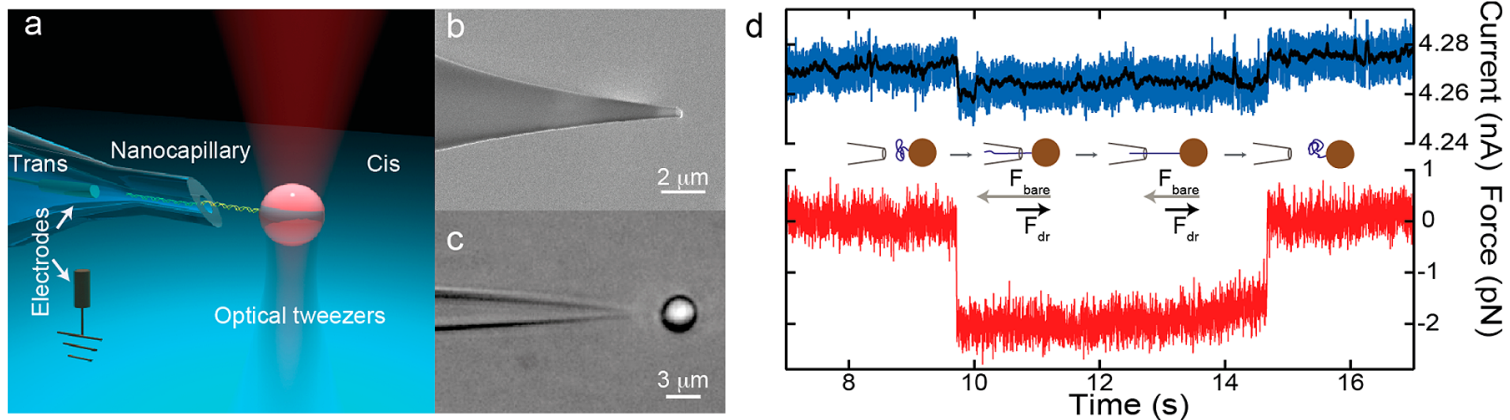

Figure 1. Representation of the experimental setup, combining nanocapillaries with optical tweezers. (a) Schematic of the setup (not to scale). A DNA-coated bead is optically trapped, and after application of positive voltage to the trans chamber, a DNA molecule is stalled inside a nanocapillary. (b) An SEM image of a pulled nanocapillary. (c) An image of an optically trapped DNA-coated bead positioned in front of a nanocapillary. (d) Data from a typical experiment in which a single DNA molecule is inserted inside a nanocapillary, followed by reverse translocation of the DNA. The simultaneous change of the current and the stalling force corresponds to the capturing of a DNA molecule. After the $12^{\text {th }} \mathrm{s}$, reverse translocation of the DNA is performed using a nanopositioning stage with a velocity of $250 \mathrm{~nm} / \mathrm{s}$. The simultaneous restoring of the stalling force and the current to their initial levels corresponds to the extraction of a single DNA molecule from the nanocapillary. The schematic represents the consecutive steps of the experiment. The arrows show the direction of the bare electrostatic force $\left(F_{\text {bare }}\right)$ and the drag force $\left(F_{\mathrm{dr}}\right)$ simultaneously acting on DNA. The experiment was conducted with a $32 \mathrm{~nm}$ nanocapillary in $1 \mathrm{M} \mathrm{KCl}, \mathrm{pH} 8.7$, at $100 \mathrm{mV}$ using a $5.5 \mathrm{~kb}$ DNA fragment.
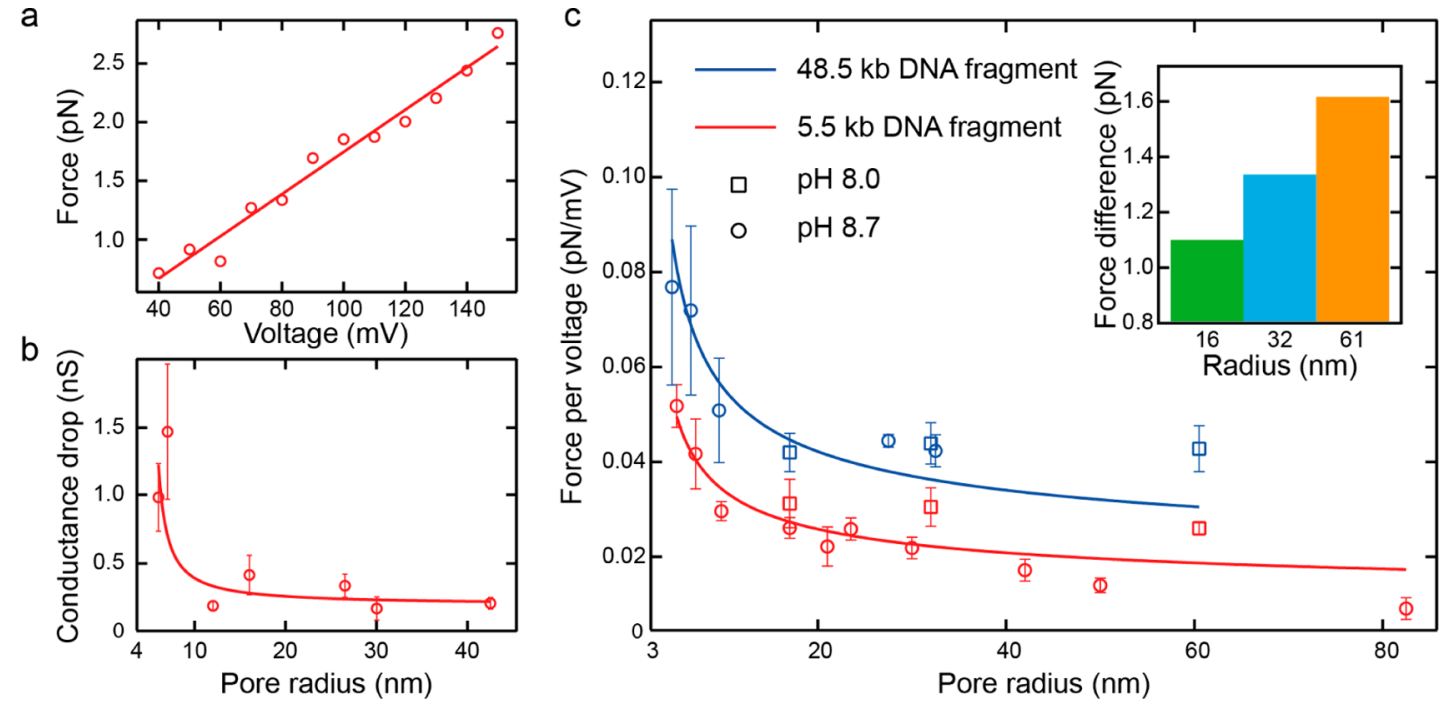

Figure 2. Investigation of the stalling force and conductance drop in nanocapillaries of different sizes. (a) Stalling force as a function of applied potential. The data were acquired with a $53 \mathrm{~nm}$ nanocapillary, in $1 \mathrm{M} \mathrm{KCl}, \mathrm{pH} 8.7$, using the $5.5 \mathrm{~kb} \mathrm{DNA}$ fragment. The data were fit to linear function. (b) Measured conductance drop versus nanocapillary radius in $1 \mathrm{M} \mathrm{KCl}, \mathrm{pH} 8.7$, using the $5.5 \mathrm{~kb}$ DNA fragment. Data were fit to eq 3 from ref 23. (c) Measured stalling force versus nanocapillary radius. The data were acquired in $40 \mathrm{mM} \mathrm{KCl}, \mathrm{pH} 8.7$ (circles) or $\mathrm{pH} 8.0$ (squares) for the $5.5 \mathrm{~kb}$ DNA fragment (red), and $\lambda$-DNA $(48.5 \mathrm{~kb})$ (blue). The data were fit to eq 2 . The length of DNA inside the nanocapillary varied from $0.4-1.35 \mu \mathrm{m}$ for the $5.5 \mathrm{~kb}$ DNA fragment and $\approx 12-14.5 \mu \mathrm{m}$ for $\lambda$-DNA. The inset shows the difference in the stalling force for the $\lambda$-DNA and 5.5 $\mathrm{kb}$ DNA fragments in $40 \mathrm{mM} \mathrm{KCl}, \mathrm{pH} 8.0$ (see text for details). The stalling force acting on the longer DNA was higher, and the difference is represented in $\mathrm{pN}$.

as a powerful tool for single molecule studies and have achieved the resolution level of solid-state nanopores.

Due to the shape of a nanocapillary, the electrophoretic driving force acting on DNA depends on its position inside the nanocapillary. This geometry results in a different velocity of molecules depending on the distance from the opening. ${ }^{25}$ In this work, we investigated how the geometry of nanocapillaries affects DNA molecules trapped inside. Our objective was to characterize the position-dependent electrophoretic force on DNA inside shrunken nanocapillaries of different sizes.

Results and Discussion. During voltage-driven translocation through a nanocapillary, a DNA molecule experiences an electrophoretic force $\left(F_{\mathrm{ep}}\right)$ consisting of the bare electrostatic force $\left(F_{\text {bare }}\right)$ opposed by the drag force $\left(F_{\mathrm{dr}}\right):{ }^{26}$

$$
F_{\text {ep }}=F_{\text {bare }}-F_{\text {dr }}
$$

$F_{\mathrm{dr}}$ originates from the electroosmotic flow of counterions that screen the negatively charged DNA backbone and glass walls of the nanocapillary. In order to measure the electrophoretic force acting on DNA inside nanocapillaries, we used optical tweezers and DNA-coated beads as a force transducer (Figure 1a). The force obtained in such a system-the stalling force-is in balance with the electrophoretic force $F_{\text {stal }}=-F_{\text {ep }}$.

The glass nanocapillaries were fabricated by simultaneous heating and pulling of capillaries as described in ref 15 and were imaged under SEM. The typical side profile of a nanocapillary is shown in Figure 1b. We shrank the pulled capillaries down to the sizes ranging from 9 to $165 \mathrm{~nm}$ in diameter by exposing them to electron irradiation. ${ }^{22}$ The shrunken nanocapillaries 

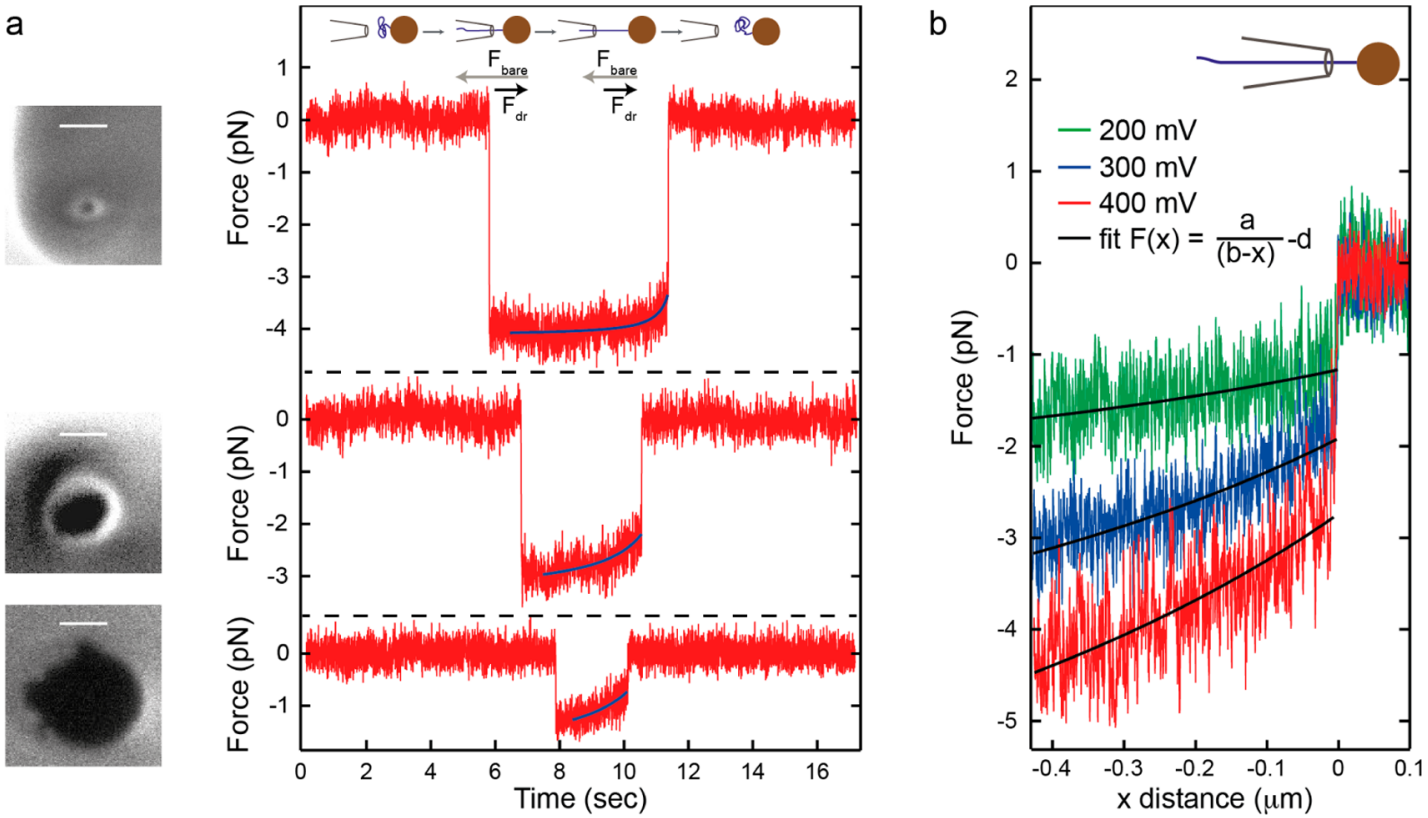

Figure 3. Mapping the gradients of stalling force inside nanocapillaries of different sizes and at different voltages. (a) Stalling force profiles for nanocapillaries with diameters of 14, 47, and $100 \mathrm{~nm}$ (from top to bottom). SEM images of the nanocapillaries are shown on the left (scale bars, 50 $\mathrm{nm})$. The schematic represents the consecutive steps of the experiment. The arrows show the direction of the bare electrostatic force $\left(F_{\text {bare }}\right)$ and the drag force $\left(F_{\mathrm{dr}}\right)$ simultaneously acting on DNA. The experiments were performed in $40 \mathrm{mM} \mathrm{KCl}, \mathrm{pH} 8.7$, at $100 \mathrm{mV}$, using the $5.5 \mathrm{~kb} \mathrm{DNA}$, and a stage velocity of $250 \mathrm{~nm} / \mathrm{s}$. The electrophoretic force profiles recorded during activation of the nanopositioning stage were fit to eq 4, where coefficient $b$ was fixed in accordance with the opening radius, and coefficients $a$ and $d$ were used as fitting parameters. (b) Measured stalling forces as a function of the distance inside a nanocapillary recorded at 200,300 , and $400 \mathrm{mV}$. The schematic shows the orientation of the nanocapillary. The experiments were performed with the $165 \mathrm{~nm}$ nanocapillary in $40 \mathrm{mM} \mathrm{KCl}, \mathrm{pH} 8.7$, using the $5.5 \mathrm{~kb}$ DNA fragment.

were placed in a sample cell, where they connected cis and trans chambers filled with buffers containing either $40 \mathrm{mM}$ or 1 $\mathrm{M} \mathrm{KCl}$. Streptavidin-coated beads were modified with either biotinylated $48.5 \mathrm{~kb}(16.5 \mu \mathrm{m}) \lambda$-DNA or a biotinylated $5.5 \mathrm{~kb}$ $(1.85 \mu \mathrm{m})$ DNA PCR fragment.

In a typical experiment, we optically trapped a bead and positioned its surface $3-4 \mu \mathrm{m}$ from the capillary opening. Next, we applied a positive potential of $50-150 \mathrm{mV}$ in the trans chamber. The voltage attracted DNA molecules attached to the optically trapped bead and forced the bead to displace toward the capillary opening. This displacement was measured using a position sensitive detector (see Materials and Methods for details). If we did not observe capture of the DNA within $90 \mathrm{~s}$, we decreased the distance between the bead and the tip by using a piezoelectric nanopositioning system. Normally, the $\lambda$ DNA capturing events took place at a distance of $1.5-3.5 \mu \mathrm{m}$ from the bead surface to the capillary opening, whereas in the case of the $5.5 \mathrm{~kb}$ DNA fragment we had to decrease the distance down to $0.5-1.5 \mu \mathrm{m}$. This observation can be explained by the difference in gyration radius of these DNA fragments ${ }^{27}$ and was previously demonstrated. ${ }^{28}$ The translocation of a DNA molecule attached to a bead resulted in a simultaneous change in the stalling force and nanocapillary conductance (Figure 1d). Controllable insertion of only one molecule inside a glass nanocapillary was achieved by adjusting the number of DNA molecules on the bead surface, the distance between the beads and glass nanopores, and the applied voltage. After stalling DNA inside a nanocapillary, reverse translocation of the DNA was performed by using the piezoelectric nanopositioning stage at a velocity of $100-500$ $\mathrm{nm} / \mathrm{s}$ with a single step of $1.5 \mathrm{~nm}$ (Movies in the Supporting Information). At the distance corresponding to the length of
DNA, we observed restoration of the conductance and stalling force to their initial levels (Figure 1d).

During the first stage of this study, we investigated the magnitude of the stalling force as a function of the size of the nanocapillary and the length of the DNA handle. After capturing a single DNA molecule, we ensured that in the range of $40-150 \mathrm{mV}$ the stalling force increases linearly with the voltage (Figure $2 \mathrm{a}$ ). In the next step, we investigated the magnitude of the conductance and stalling force drops caused by inserting a DNA molecule in nanocapillaries of different diameters. In $1 \mathrm{M} \mathrm{KCl}$ solution, the conductance change was higher for the nanocapillaries of smaller diameters (Figure 2b) in accordance with ref 23. However, in $1 \mathrm{M} \mathrm{KCl}$ solution sometimes we observed sticking of the DNA to glass walls inside the nanocapillary (data not shown). The repulsion between two negatively charged surfaces of DNA and glass can be decreased due to the significant effect of screening at high salt concentration. ${ }^{29}$ On the other hand, in the buffer with low ionic strength $(40 \mathrm{mM} \mathrm{KCl})$, we did not observe nonspecific interactions of DNA with glass walls. This is a promising result, particularly in the context of experiments that aim to probe protein-DNA interactions and thus require low ionic strength. In $40 \mathrm{mM} \mathrm{KCl}$ solution the stalling force increases with decreasing of nanocapillary size (Figure 2c, Movies S1 and S2), which is consistent with solid-state nanopores. ${ }^{8}$ The negatively charged backbone of DNA trapped inside a nanocapillary induces an opposite flow of counterions, resulting in additional drag force on the DNA molecule. The drag force depends on the nanopore diameter and is of smaller magnitude in smaller nanopores. We fit the stalling forces as a function of a nanocapillary opening by using the equation: 

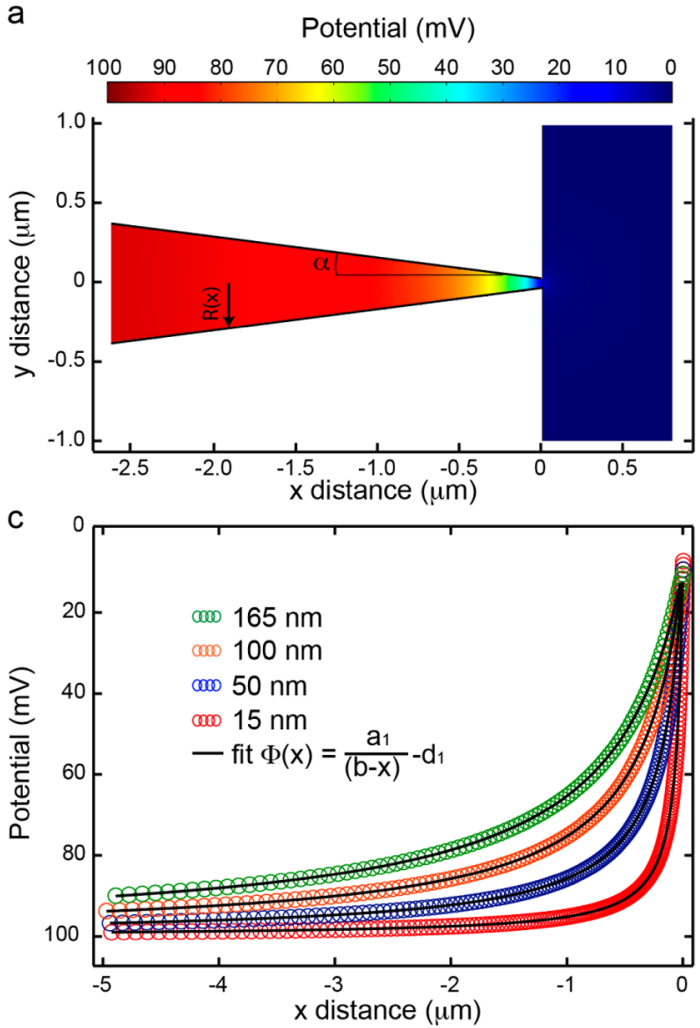

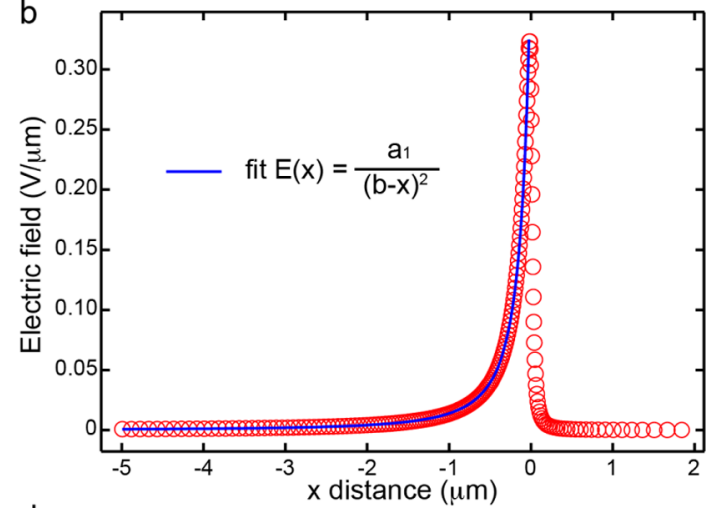

d

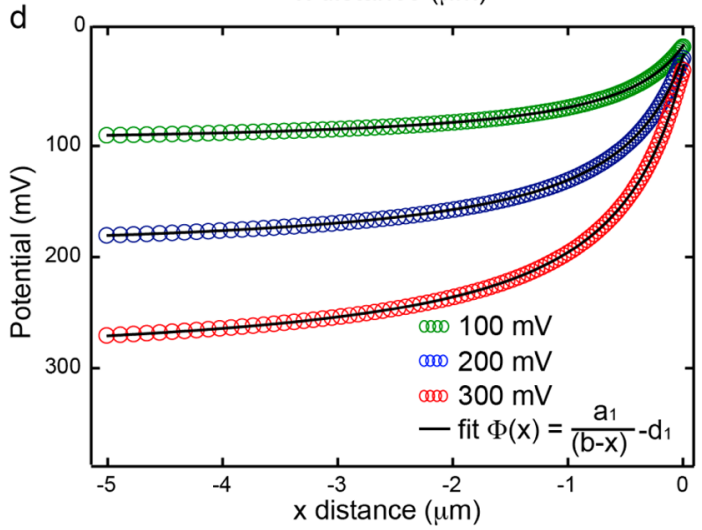

Figure 4. Modeling of the position-dependent potential and electric field in nanocapillaries. (a) Finite element analysis of the position-dependent potential in the conical nanocapillary with an opening radius $R_{0}=30 \mathrm{~nm}$ and an opening angle $\tan \alpha=0.133$. The modeling was performed in 40 $\mathrm{mM} \mathrm{KCl}$ at $100 \mathrm{mV}$. (b) The position-dependent electric field for the nanocapillary shown in a. The data were fit to eq 7, and coefficient $b$ was fixed in accordance with the radius of the nanocapillary and using $a_{1}$ as a fitting parameter. (c) The position-dependent potentials in nanocapillaries with diameters of $15,50,100$, and $165 \mathrm{~nm}$, calculated in $40 \mathrm{mM} \mathrm{KCl}$ at $100 \mathrm{mV}$. The data were fit to eq 8 , and coefficient $b$ was fixed in accordance with the radius of the nanocapillary, using coefficients $a_{1}$ and $d_{1}$ as fitting parameters. (d) The position-dependent potentials for a $165 \mathrm{~nm}$ nanocapillary, calculated at 100,200, and $300 \mathrm{mV}$. Data were fit to eq 8, and coefficient $b$ was fixed in accordance with the radius of the nanocapillary, coefficients $a_{1}$ and $d_{1}$ as fitting parameters. All fitting parameters are shown in Table S1.

$$
F(R) \sim \frac{1}{\ln (R / 1.1)}
$$

where $R$ is the radius of a nanocapillary and 1.1 represents the radius of a dsDNA in $n m .{ }^{8,20,30}$ However, the stalling forces in nanocapillaries were smaller than those in solid-state nanopores $^{8,9,31}$ (Figure 2c). To explain this observation, a nanocapillary can be considered as a sequence of ultrathin nanopores with continuously increasing diameters. The increase in the size of the pore will lead to an increase in the drag force, as mentioned before, resulting in a smaller total stalling force. Another aspect affecting the magnitude of the stalling force is the electroosmotic flow acting on DNA, which is caused by the negatively charged glass walls. The presence and characterization of an electroosmotic nanojet produced by the glass walls of nanocapillaries have been previously shown by calculating the rotation frequency of slightly asymmetric beads trapped in front of the openings. ${ }^{21}$ The effect of electroosmotic flow on DNA translocation was also demonstrated for solidstate nanopores. ${ }^{31}$ In this recent work the modification of a pore surface with neutrally charged lipids almost doubled the magnitude of the stalling force.

In addition to the diameter of nanocapillaries, DNA length also influenced the magnitude of the stalling force. In general, the stalling forces for $48.5 \mathrm{~kb} \lambda$-DNA were higher than those for the $5.5 \mathrm{~kb}$ DNA fragment (Figure 2c). For the nanocapillaries with diameters of 32,64 , and $121 \mathrm{~nm}$, we first measured the stalling force acting on the $5.5 \mathrm{~kb}$ DNA fragment. Afterward we removed the DNA-coated beads by rinsing the cis chamber with a $40 \mathrm{mM} \mathrm{KCl}$ buffer. In the next step $\lambda$-DNA-coated beads were injected, and we were able to measure the stalling force acting on longer $\lambda$-DNA molecules.

We observed that the stalling force on the $\lambda$-DNA was higher, especially for nanocapillaries exceeding $60 \mathrm{~nm}$ in diameter (Figure 2c, inset). The length-dependent behavior of the stalling force can be attributed to the fact that for shorter DNA the optically trapped bead has to be positioned closer to the opening and consequently it experiences higher electroosmotic flow. ${ }^{21}$ In addition, for longer DNA the increased number of basepairs inside the nanocapillary leads to the higher bare electrostatic force $\left(F_{\text {bare }}\right)$ due to the extended electric field. A more detailed explanation will be discussed later.

The dependence of the stalling force on the length of the DNA handle prompted us to continuously vary the length of captured DNA inside the nanocapillaries by performing reverse translocation. This method, implemented on the $5.5 \mathrm{~kb}$ DNA fragment, allowed us to measure the DNA length trapped inside the nanocapillary (Figure S1) and to record the positiondependent stalling force profiles (later in the terms "positiondependent force profiles/force profiles/force decays", the word "stalling" is omitted for simplicity). The profiles varied in magnitude and shape with the size of the nanocapillaries (Figure 3a). In the case of nanocapillaries less than $20 \mathrm{~nm}$ in diameter, the force decay was insignificant and took place only 
in the final part of the nanocapillaries, close to the opening. This profile closely resembles the data reported for solid-state nanopores, where the stalling force is kept constant along the entire nanopore length. ${ }^{11,31}$ However, the increase of the nanocapillary size leads to longer decays of the force profiles. For example, for the $100 \mathrm{~nm}$ nanocapillary the decay in the stalling force was pronounced along the entire length of DNA (Figure 3a). There was no visible difference between the profiles recorded in the same nanocapillary for the $5.5 \mathrm{~kb}$ DNA and $\lambda$-DNA (Figure S2). The smaller stalling force acting on shorter DNA could be attributed to the already mentioned higher electroosmotic flow on a bead, heating effects of the laser and small uncertainties in the force calibration regarding the trap stiffness.

In addition to the size-dependent behavior in the range of diameters of 9-165 nm, we investigated the influence of applied voltage on the position-dependent force profiles. We pulled the DNA molecule out of the $165 \mathrm{~nm}$ nanocapillary under applied voltages of 200, 300, and $400 \mathrm{mV}$ (Figure 3b). We observed a variation in the slope of the position-dependent force at different applied voltages.

To provide an explanation for the size- and voltagedependent behavior of the force profiles, we performed analytical and numerical calculations. Based on ref 7, the position-dependent force $F(x)$ inside a nanocapillary is proportional to the position-dependent potential $\Phi(x)$ :

$$
F(x)=-\frac{q_{\text {eff }}}{l} \Phi(x)
$$

where $q_{\text {eff }}$ is the effective charge of a DNA base pair and $l$ represents the distance between two base pairs. We modeled $\Phi(x)$ and $E(x)$ inside a nanocapillary with finite element analysis using COMSOL Multiphysics software. We represented a nanocapillary as a cone with an opening angle $\tan \alpha=$ 0.133 (Figure $4 \mathrm{a}$ ), and varying radii $R_{0}$. A typical profile of the position-dependent electric field $E(x)$ in the $60 \mathrm{~nm}$ nanocapillary is shown in Figure $4 \mathrm{~b}$. We compared the distancedependent potentials $\Phi(x)$ extracted from the finite element model for 15, 50, 100, and $165 \mathrm{~nm}$ nanocapillaries. The $\Phi(x)$ profiles were steeper for smaller nanocapillaries. Almost $90 \%$ of the applied potential drops were within the first micrometer from the opening inside the $15 \mathrm{~nm}$ nanocapillary, whereas for the $165 \mathrm{~nm}$ nanocapillary this value was only $\approx 60 \%$. This finding explains the difference in the position-dependent force profiles for nanocapillaries of different sizes (Figure 3a) and is one of the reasons for the higher stalling force exerted on the $48.5 \mathrm{~kb}$ DNA versus $5.5 \mathrm{~kb}$ DNA (Figure 2c). Since the component of the electroctrophoretic force acting on a tethered DNA molecule from the outside of a nanocapillary is much less than from the inside, it was not taken into account in the following analysis (Figure S3).

From the analytical model (Materials and Methods) we concluded that $E(x)$ inside nanocapillaries decays proportionally to $\mathrm{x}^{-2}$ (eq 7), whereas $\Phi(x)$ obeys simple hyperbolic behavior (eq 8). To validate the finite element analysis simulations, we fit $E(x)$ and $\Phi(x)$ acquired in COMSOL to eqs 7 and 8 of Materials and Methods as shown in Figure 4b, c, and $\mathrm{d}$. We observed an almost perfect fit of the COMSOL results to the analytical model.

Based on eq 3 and eq 8 from Materials and Methods, the position-dependent force can be described by a hyperbolic expression:

$$
F(x)=\frac{a}{b-x}-d
$$

where $a, b$, and $d$ are parameters that do not depend on the distance $x$ inside a nanocapillary. Equation 4 represents a hyperbolic function, where coefficients $b$ and $d$ correspond to horizontal and vertical displacements of the curve, respectively, and coefficient $a$ describes the slope of the curve (Figure S4). Equation 4 demonstrates that the electrophoretic force acting on different parts of a translocating DNA molecule decays with the distance inside the nanocapillary. The portion of the DNA closest to the nanocapillary opening experiences higher electrophoretic force than the part that is already further away from the entrance. Moreover, the electrophoretic force decay depends on the geometry of the nanocapillary, and it is steeper for nanocapillaries of smaller diameters. While coefficient $d$ in eq 4 is responsible for the magnitude of the stalling force, coefficients $a$ and $b$ describe the behavior of the decay of the electrophoretic force on DNA with the distance inside nanocapaillaries of different sizes.

We fit our experimental data for nanocapillaries of different sizes to eq 4. The results are shown in Table 1 . Coefficient $b$,

Table 1. Coefficients Used To Describe the PositionDependent Force Profiles for Nanocapillaries of Different Sizes ${ }^{a}$

\begin{tabular}{cccc} 
& \multicolumn{3}{c}{ coefficients } \\
\cline { 2 - 4 } pore radius $(\mathrm{nm})$ & $a(\mathrm{pN} \cdot \mu \mathrm{m})$ & $b(\mu \mathrm{m})$ & $d(\mathrm{pN})$ \\
5 & $0.03 \pm 0.0003$ & 0.038 & $5.01 \pm 0.001$ \\
7 & $0.037 \pm 0.001$ & 0.05 & $4.08 \pm 0.002$ \\
23.5 & $0.17 \pm 0.002$ & 0.18 & $2.86 \pm 0.004$ \\
30 & $0.19 \pm 0.002$ & 0.23 & $2.32 \pm 0.004$ \\
42.5 & $0.27 \pm 0.003$ & 0.33 & $1.74 \pm 0.01$ \\
50 & $0.33 \pm 0.004$ & 0.38 & $1.55 \pm 0.01$ \\
82.5 & $0.49 \pm 0.01$ & 0.63 & $1.16 \pm 0.01$
\end{tabular}

${ }^{a}$ The force profiles were fit to eq 4 , where coefficient $b$ was fixed in accordance with the radius of a nanocapillary, and coefficients $a$ and $d$ were used as fitting parameters. The data were acquired in $40 \mathrm{mM}$ $\mathrm{KCl}, \mathrm{pH} 8.7$, at $100 \mathrm{mV}$.

which represents the size of the capillary opening (see Materials and Methods for details), was estimated from SEM images. During the fitting procedure, coefficient $b$ was fixed, and only coefficients $a$ and $d$ were used as fitting parameters. We noticed a trend of increasing of coefficient $a$ values for larger nanocapillaries. Coefficient $a$ allows for the quantitative comparison of force profile slopes for nanocapillaries of different diameters. In contrast, coefficient $d$ value decreased when the nanocapillary size was increased, due to the dependence of the stalling force on the opening size (Figure 2c). In addition, we analyzed the voltage-dependent behavior of force profiles inside a $165 \mathrm{~nm}$ nanocapillary using the same approach. The data were fit to eq 4 and represented in Table 2. We observed that the absolute values of coefficients $a$ and $d$ increased almost linearly at higher applied voltages.

However, there are some simplifications in our model that we would like to emphasize below. First of all, the geometry of a laser pulled nanocapillary deviates from the shape of an ideal cone (Figure 1b). ${ }^{23}$ Shrinking of nanocapillaries under SEM leads to an additional change of the shape close to the tip, especially for small nanocapillaries. Moreover, the proposed model does not explicitly take into account the drag force $\left(F_{\mathrm{dr}}\right)$ on DNA and relies only on the bare electrostatic force $\left(F_{\text {bare }}\right)$ 
Table 2. Coefficients Used To Describe the PositionDependent Force Profiles for Nanocapillaries at Different Voltages $^{a}$

\begin{tabular}{cccc} 
& \multicolumn{3}{c}{ coefficients } \\
\cline { 2 - 4 } voltage $(\mathrm{mV})$ & $a(\mathrm{pN} \cdot \mu \mathrm{m})$ & $b(\mu \mathrm{m})$ & $d(\mathrm{pN})$ \\
200 & $0.93 \pm 0.01$ & 0.63 & $2.55 \pm 0.02$ \\
300 & $2.05 \pm 0.01$ & 0.63 & $5.13 \pm 0.01$ \\
400 & $2.97 \pm 0.02$ & 0.63 & $7.38 \pm 0.02$
\end{tabular}

${ }^{a}$ The force profiles were fit to eq 4 , where coefficient $b$ was fixed in accordance with the radius of a nanocapillary, and coefficients $a$ and $d$ were used as fitting parameters. The data were acquired in $40 \mathrm{mM}$ $\mathrm{KCl}, \mathrm{pH} 8.7$, for the $165 \mathrm{~nm}$ nanocapillary.

through the assumption of a constant effective charge. Nevertheless, our model provides a good qualitative explanation of the experimental results and enables comparison of the force profile slopes recorded in nanocapillaries of different sizes and at different voltages.

In this work we investigated the electrophoretic force on DNA in glass nanocapillaries shrunken under SEM. We demonstrated that the electrophoretic position-dependent force profiles depend on the nanocapillary size and the applied voltage. The results of this work provide a better understanding of the translocation behavior of charged molecules through nanocapillaries. Compared to nanopores in a membrane, conical glass nanopores represent a system that can be easily tailored to simultaneously apply forces of different magnitude on different parts of tethered biomolecules. This type of force measurement may be especially useful in probing DNAprotein complexes and intramolecular interactions.

Materials and Methods. Nanocapillaries. Quartz capillaries with a $0.3 \mathrm{~mm}$ inner diameter and a $0.4 \mathrm{~mm}$ outer diameter (Hilgenberg) were pulled using a P-2000 laser pipet puller (Sutter) and one of two programs depicted in Table 3.

Table 3. Pulling Parameters of the Pipette Puller P-2000 for the Fabrication of Nanocapillaries of 100-150 nm (Program 1) and 200-300 nm (Program 2) Diameter

\begin{tabular}{cccccc} 
program & heat & filament & velocity & delay & pull \\
1 & 620 & 0 & 30 & 140 & 200 \\
2 & 580 & 0 & 30 & 140 & 120 \\
\hline
\end{tabular}

The first program resulted in openings of $100-150 \mathrm{~nm}$ with a pulling time of $0.30 \pm 0.02 \mathrm{~s}$. The second program resulted in openings of $200-300 \mathrm{~nm}$ with a pulling time of $0.47 \pm 0.03 \mathrm{~s}$. The pulled nanocapillaries were imaged under scanning electron microscope Merlin SEM (Zeiss). Continuous imaging of nanocapillaries for $0.5-5 \mathrm{~min}$ resulted in shrinking of the nanocapillaries to the desired diameters $(9-165 \mathrm{~nm})$. The working distance during the imaging was $2.5-5 \mathrm{~mm}$, magnification 80-150×, beam current 500-550 pA, and acceleration voltage $2-3 \mathrm{kV}$. Sometimes shrinking did not result in nanocapillaries with a circular shape of the opening. In this case the size was extracted based on measuring the surface area using ImageJ. The opening angle $(\alpha)$ of nanocapillaries was estimated from both optical microscopy and SEM of the tip (Figure $1 \mathrm{~b}$ ). The value $\tan \alpha=0.133$ was used for fitting the position-dependent force profiles.

The shrunken nanocapillaries were put inside a PDMS sample cell, in order to connect the cis and trans chambers only through the nanocapillary opening. The cell was sealed from the bottom with $0.15 \mathrm{~mm}$ thick coverslip (Menzel-Glässer). To make the surface of PDMS and glass hydrophilic, the sample cells were treated with oxygen plasma at the power $50 \mathrm{~W}$ for 3-5 min. Then the chambers were filled with a buffer containing $40 \mathrm{mM}$ or $1 \mathrm{M} \mathrm{KCl}$, with $3 \mathrm{mM}$ Tris/boric acid, $\mathrm{pH} 8.7$, or $3 \mathrm{mM}$ Tris/ $\mathrm{HCl}, \mathrm{pH} 8.0$, and $1 \mathrm{mM}$ or $25 \mathrm{mM}$ EDTA. Such a high concentration of EDTA served to decreasing the interactions of DNA with a glass surface. ${ }^{32}$ Before an experiment, air bubbles inside the nanocapillaries were removed by evacuating them with a vacuum pump for $1-$ 5 min.

Setup. The setup consists of optical tweezers and current preamplifier (Figure S5). The laser beam, generated by a $\mathrm{Nd}$ YVO4 solid-state laser (Coherent, $2.5 \mathrm{~W}, \lambda=1064 \mathrm{~nm}$ ) and expanded by a telescope $(2.5 \times)$, forms the steep gradient after passing the water immersion microscope objective (Nikon, $60 \times, 1.2 \mathrm{NA}$ ). The transmitted light is collected by a condenser (Olympus, $0.8 \mathrm{NA}$ ) and deflected onto a photosensitive detector (Pacific Silicon Sensor Inc.). A sample cell is positioned on a piezoelectric nanopositioning stage (Mad City Laboratories) and illuminated by a white light source (Thorlabs). An image is formed on a CCD camera (Thorlabs). $\mathrm{Ag} / \mathrm{AgCl}$ electrodes inserted in cis and trans chambers of the sample cell and connected to an Axopatch 200B current amplifier (Molecular Devices), used for applying the potential and measuring the ionic current. The sample cell is enclosed in an aluminum Faraday cage, which blocks the influence from external electric fields. The ionic current and signals from the photosensitive detector were recorded using custom-made LabVIEW software.

DNA-Coated Beads. In order to make DNA-coated beads, we incubated streptavidin-coated beads (Bangs Laboratories) with DNA molecules biotinylated from one end. Biotinylated $48.5 \mathrm{~kb} \lambda$-DNA and $5.5 \mathrm{~kb}$ DNA fragments were used in this work. To attach a biotin tag to $\lambda$-DNA (New England BioLabs), we ligated it with a $3^{\prime}$-end biotinylated oligonucleotide complementary to the $\cos 2$ overhang of $\lambda$-DNA. ${ }^{33}$ To synthesize the $5.5 \mathrm{~kb}$ DNA fragment biotinylated from one end, we performed PCR of genomic DNA of phage T7 with Tag polymerase using two primers: 5'-ATC GAC CCT GAG GAA CTC ATC-3' and (B)-5'-CAC CAT(B) CT(B)A ACA GTC CCA TCA G-3'. Modifications of nucleotides with biotin are marked with (B). The Macherey-Nagel purification kit was used for cleaning of the PCR product. We incubated $3 \mu \mathrm{m}$ streptavidin-coated beads with the DNA construct in the ratio 100-1000 DNA molecules per 1 bead in the buffer, containing $100 \mathrm{mM} \mathrm{KCl}, 10 \mathrm{mM}$ Tris$/ \mathrm{HCl}, \mathrm{pH} 7.0$ during 60-90 min. Then we washed them 2-3 times in the incubation buffer to remove nonbound DNA molecules and resuspended in the buffer used in experiments. A sample of $1-3 \mu \mathrm{L}$ of $0.2 \mathrm{mg} / \mu \mathrm{L}$ DNA-coated beads was added to the buffer in cis chamber, and the solution was mixed. In some experiments we noticed that $\lambda$ DNA was subject to shear stress, most likely due to pipetting or freeze-thawing cycles, and the observed length was around 4 or $8 \mu \mathrm{m}$ instead of $16.5 \mu \mathrm{m}$. $^{34}$

Calibration of Optical Tweezers. The optical tweezers were calibrated using the power spectrum density method and Stokes methods. ${ }^{35}$ In the first case the thermal fluctuations of a $3 \mu \mathrm{m}$ DNA-coated trapped bead were recorded at $500 \mathrm{kHz}$ during 5-10 s, and afterward the cutoff frequency was extracted (Figure S6a). The presence of DNA molecules on a bead increases the friction coefficient of beads and consequently decreases cutoff frequency (Figure S6b). The difference in 
cutoff frequency for DNA-coated beads versus noncoated beads shown in Figure S6b was used for correction of stiffness estimated in experiments. The Stokes method was carried out on DNA-coated beads using sinusoidal oscillations of the nanopositioning stage. The calibration procedure was performed on at least five different beads and at least $20 \mu \mathrm{m}$ away from any surface. The stiffness estimated by these two methods did not vary by more than $20 \%$. In a typical experiment the stiffness was set in the range of $9-20 \mathrm{pN} / \mu \mathrm{m}$, which roughly corresponded to $9-20 \mathrm{~mW}$ laser power at sample plane. In case of nanocapillaries less than $20 \mathrm{~nm}$ in diameter and $\lambda$-DNA as a handle, the stiffness was set to $109 \mathrm{pN} / \mu \mathrm{m}$ that facilitated the experiments. Measurements of the sensitivity of the response of photosensitive detector $(\mathrm{V} / \mu \mathrm{m})$ were performed on a $3 \mu \mathrm{m}$ bead stuck to a cover slide and repeated at least on five different beads (Figure S6c).

Finite Element Analysis. The numerical simulations of the position-dependent potential and electric field were performed in COMSOL $4.3 \mathrm{~b}$ Multiphysics. We generated the 3D geometry with an axial symmetry and solved the PoissonNernst-Planck (PNP) equation using finite element calculations with minimum mesh elements of less than $0.5 \mathrm{~nm}$. The nanocapillaries were modeled as a cone with an opening angle $\tan \alpha=0.133$ (Figure 4a). The radius of the opening varied in the range of $5-82.5 \mathrm{~nm}$ and the applied voltage in the range of $100-300 \mathrm{mV}$. The calculations were carried out in $40 \mathrm{mM} \mathrm{KCl}$ solution. The surface charge of a nanocapillary wall in the model was set to zero.

Analytical Calculations. According to ref 36 and neglecting the small influence of the DNA molecule captured inside a nanocapillary, the position-dependent electric field $E(x)$ can be related to the ionic current $I$ :

$$
E(x)=\frac{I}{\sigma \pi R(x)^{2}}
$$

where $\sigma$ is the specific conductivity of the solution and $R(x)$ is the position-dependent radius of a nanocapillary. Taking into account that for a nanocapillary with conical shape $R(x)=R_{0}$ $-x \tan \alpha$ (Figure 4a, please note that $x$ values are negative), where $R_{0}$ is the radius of a nanocapillary opening, $\alpha$ is the opening angle of a nanocapillary (Figure $4 \mathrm{a}$ ), the positiondependent electric field can be expressed as

$$
E(x)=\frac{I}{\sigma \pi \tan ^{2} \alpha\left(R_{0} / \tan \alpha-x\right)^{2}}
$$

or

$$
E(x)=\frac{a_{1}}{(b-x)^{2}}
$$

where $a_{1}=I / \sigma \pi \tan ^{2} \alpha$ and $b=R_{0} / \tan \alpha$. Since the potential $\Phi(x)=-\int E(x) \mathrm{d} x$, integrating of eq 7 results in

$$
\Phi(x)=\frac{a_{1}}{(b-x)}-d_{1}
$$

where $d_{1}$ is an integration constant.

\section{ASSOCIATED CONTENT}

\section{S Supporting Information}

Supplementary methods with legends and additional reference, supplementary Table S1, Figures S1-6, and Movies S1-2. This material is available free of charge via the Internet at http:// pubs.acs.org.

\section{AUTHOR INFORMATION}

\section{Corresponding Author}

*E-mail: aleksandra.radenovic@epfl.ch.

\section{Author Contributions}

R.D.B., L.J.S., and A.R. designed the study. R.D.B. and L.J.S. pulled and shrank nanocapillaries. R.D.B. carried out experiments. R.D.B. performed data analysis. S.K., J.S.S., R.D.B., and L.J.S. performed COMSOL modeling. R.D.B. wrote the paper. U.F.K. read the paper and gave valuable suggestions. A.R. supervised the project, built optical tweezers setup, and provided resources. All authors provided important suggestions for the experiments, discussed the results, and contributed to the manuscript.

\section{Notes}

The authors declare no competing financial interest.

\section{ACKNOWLEDGMENTS}

We thank Center of Micro/Nanotechnology (CMI) EPFL for access to SEM. Special thanks to Prof. Olivier Martin and Dr. Christian Santschi for providing the pipet puller. We acknowledge Fabrizia Dutto for help with optical tweezers. Tania Palmieri for pulling nanocapillaries. Oliver Otto, Fabrizia Dutto, Ke Liu, Kunal Sharma, and Kristina Woodruff for valuable discussion. Camille Raillon and Serena Brando for initial help with the setup. This work was financially supported by the European Research Council (grant no. 259398, PorABEL: Nanopore integrated nanoelectrodes for biomolecular manipulation and design).

\section{REFERENCES}

(1) Li, J.; Gershow, M.; Stein, D.; Brandin, E.; Golovchenko, J. A. Nat. Mater. 2003, 2 (9), 611-5.

(2) Skinner, G. M.; van den Hout, M.; Broekmans, O.; Dekker, C.; Dekker, N. H. Nano Lett. 2009, 9 (8), 2953-2960.

(3) Han, A. P.; Schurmann, G.; Mondin, G.; Bitterli, R. A.; Hegelbach, N. G.; de Rooij, N. F.; Staufer, U. Appl. Phys. Lett. 2006, 88 (9), 093901.

(4) Raillon, C.; Cousin, P.; Traversi, F.; Garcia-Cordero, E.; Hernandez, N.; Radenovic, A. Nano Lett. 2012, 12 (3), 1157-64.

(5) Venkatesan, B. M.; Bashir, R. Nat. Nanotechnol. 2011, 6 (10), 615-24.

(6) Wanunu, M. Phys. Life Rev. 2012, 9 (2), 125-58.

(7) Keyser, U. F.; Koeleman, B. N.; Van Dorp, S.; Krapf, D.; Smeets, R. M. M.; Lemay, S. G.; Dekker, N. H.; Dekker, C. Nat. Phys. 2006, 2 (7), 473-477.

(8) van Dorp, S.; Keyser, U. F.; Dekker, N. H.; Dekker, C.; Lemay, S. G. Nat. Phys. 2009, 5 (5), 347-351.

(9) van den Hout, M.; Vilfan, I. D.; Hage, S.; Dekker, N. H. Nano Lett. 2010, 10 (2), 701-707.

(10) Hall, A. R.; van Dorp, S.; Lemay, S. G.; Dekker, C. Nano Lett. 2009, 9 (12), 4441-4445.

(11) Sischka, A.; Spiering, A.; Khaksar, M.; Laxa, M.; Konig, J.; Dietz, K. J.; Anselmetti, D. J. Phys.: Condens. Matter 2010, 22 (45), 454121.

(12) Spiering, A.; Getfert, S.; Sischka, A.; Reimann, P.; Anselmetti, D. Nano Lett. 2011, 11 (7), 2978-82.

(13) Storm, A. J.; Chen, J. H.; Ling, X. S.; Zandbergen, H. W.; Dekker, C. Nat. Mater. 2003, 2 (8), 537-540.

(14) Li, J.; Stein, D.; McMullan, C.; Branton, D.; Aziz, M. J.; Golovchenko, J. A. Nature 2001, 412 (6843), 166-9.

(15) Steinbock, L. J.; Otto, O.; Chimerel, C.; Gornall, J.; Keyser, U. F. Nano Lett. 2010, 10 (7), 2493-2497.

(16) Li, W.; Bell, N. A. W.; Hernandez-Ainsa, S.; Thacker, V. V.; Thackray, A. M.; Bujdoso, R.; Keyser, U. F. ACS Nano 2013, 7 (5), 4129-4134. 
(17) Otto, O.; Steinbock, L. J.; Wong, D. W.; Gornall, J. L.; Keyser, U. F. Rev. Sci. Instrum. 2011, 82 (8), 086102.

(18) Steinbock, L. J.; Otto, O.; Skarstam, D. R.; Jahn, S.; Chimerel, C.; Gornall, J. L.; Keyser, U. F. J. Phys.: Condens. Matter 2010, 22 (45), 454113.

(19) Otto, O.; Sturm, S.; Laohakunakorn, N.; Keyser, U. F.; Kroy, K. Nat. Commun. 2013, 4, 1780.

(20) Laohakunakorn, N.; Ghosal, S.; Otto, O.; Misiunas, K.; Keyser, U. F. Nano Lett. 2013, 13 (6), 2798-802.

(21) Laohakunakorn, N.; Gollnick, B.; Moreno-Herrero, F.; Aarts, D. G. A. L.; Dullens, R. P. A.; Ghosal, S.; Keyser, U. F. Nano Lett. 2013, 13 (11), 5141-5146.

(22) Steinbock, L. J.; Steinbock, J. F.; Radenovic, A. Nano Lett. 2013, 13 (4), 1717-23.

(23) Steinbock, L. J.; Bulushev, R. D.; Krishnan, S.; Raillon, C.; Radenovic, A. Acs Nano 2013, 7, 11255-62.

(24) Steinbock, L. J.; Krishnan, S.; Bulushev, R. D.; Borgeaud, S.; Blokesch, M.; Feletti, L.; Radenovic, A. Nanoscale, 2014.

(25) Thacker, V. V.; Ghosal, S.; Hernandez-Ainsa, S.; Bell, N. A.; Keyser, U. F. Appl. Phys. Lett. 2012, 101 (22), 223704.

(26) Keyser, U. F.; van Dorp, S.; Lemay, S. G. Chem. Soc. Rev. 2010, 39 (3), 939-47.

(27) Smith, D. E.; Perkins, T. T.; Chu, S. Macromolecules 1996, 29 (4), 1372-1373.

(28) Trepagnier, E. H.; Radenovic, A.; Sivak, D.; Geissler, P.; Liphardt, J. Nano Lett. 2007, 7 (9), 2824-2830.

(29) Romanowski, G.; Lorenz, M. G.; Wackernagel, W. Appl. Environ. Microbiol. 1991, 57 (4), 1057-61.

(30) Ghosal, S. Phys. Rev. E: Stat. Nonlinear Soft Matter Phys. 2007, 76 (6 Pt 1), 061916.

(31) Galla, L.; Meyer, A. J.; Spiering, A.; Sischka, A.; Mayer, M.; Hall, A. R.; Reimann, P.; Anselmetti, D. Nano Lett. 2014, 14 (7), 41764182.

(32) Lorenz, M. G.; Wackernagel, W. Appl. Environ. Microbiol. 1987, 53 (12), 2948-52.

(33) Keyser, U. F. Methods Mol. Biol. 2012, 870, 115-34.

(34) Yoo, H.; Lim, H.; Yang, I.; Kim, S.; Park, S. J. Biophys. Chem. 2011, 2, 102-11.

(35) Neuman, K. C.; Block, S. M. Rev. Sci. Instrum. 2004, 75 (9), 2787-2809.

(36) Ghosal, S. Phys. Rev. E: Stat. Nonlinear Soft Matter Phys. 2006, 74 (4 Pt 1), 041901. 